\title{
Timelike Tangent Developable Surfaces and Bonnet Surfaces
}

\author{
Soley Ersoy and Kemal Eren \\ Department of Mathematics, Faculty of Arts and Science, Sakarya University, 54187 Sakarya, Turkey \\ Correspondence should be addressed to Soley Ersoy; sersoy@sakarya.edu.tr
}

Received 20 November 2015; Accepted 3 January 2016

Academic Editor: Chun-Gang Zhu

Copyright (C) 2016 S. Ersoy and K. Eren. This is an open access article distributed under the Creative Commons Attribution License, which permits unrestricted use, distribution, and reproduction in any medium, provided the original work is properly cited.

\begin{abstract}
A criterion was given for a timelike surface to be a Bonnet surface in 3-dimensional Minkowski space by Chen and Li, 1999. In this study, we obtain a necessary and sufficient condition for a timelike tangent developable surface to be a timelike Bonnet surface by the aid of this criterion. This is examined under the condition of the curvature and torsion of the base curve of the timelike developable surface being nonconstant. Moreover, we investigate the nontrivial isometry preserving the mean curvature for a timelike flat helicoidal surface by considering the curvature and torsion of the base curve of the timelike developable surface as being constant.
\end{abstract}

\section{Introduction}

Surfaces which admit a one-parameter family of isometric deformations preserving the mean curvature are called Bonnet surfaces. In 1867, Bonnet proved that any surface with constant mean curvature in $\mathbb{R}^{3}$ (which is not totally umbilical) is a Bonnet surface [1]. Cartan obtained some detailed results for Bonnet surfaces in [2]. Lawson extended Bonnet's results to any surface with constant mean curvature in Riemannian 3-manifold of constant curvature. Also, it was proved that any Bonnet surface of nonconstant mean curvature depends on six arbitrary constants [3]. Characterization for isometric deformation preserving the principal curvatures of surfaces was obtained by the aid of differential forms by Chern in [4]. The geometric characterizations of helicoidal surfaces of constant mean curvature, helicoidal surfaces as Bonnet surfaces, and tangent developable surfaces as Bonnet surfaces were studied by Roussos in [5], [6] and [7], respectively. Roussos obtained a characterization for isometric deformation preserving the mean curvature by using the method of Chern. Soyuçok gave the necessary and sufficient condition of a surface to be Bonnet surface, which is to have a special system of isothermal parameters [8]. Moreover, Soyuçok proved that 3-dimensional hyperspace in 4-dimensional space is Bonnet surface if and only if hypersurface has orthogonal net [9]. Bağdatlı and Soyuçok studied hypersurfaces preserving the mean curvature and proved that a hypersurface in $\mathbb{R}^{n+1}$ is Bonnet surface if and only if hypersurface has orthogonal A-net [10]. On the other hand, Chen and Li studied 3-dimensional Minkowski space and classified timelike Bonnet surfaces [11].

\section{Preliminaries}

Let $M$ be a timelike surface in 3-dimensional Minkowski space with nondegenerate metric tensor $g=-d x_{1}^{2}+d x_{2}^{2}+d x_{3}^{2}$, where $\left\{x_{1}, x_{2}, x_{3}\right\}$ is a system of the canonical coordinates in $\mathbb{R}^{3}$. Let $\Phi: M \rightarrow \mathbb{R}_{1}^{3}$ be a timelike immersion that admits a nontrivial isometry preserving the mean curvature. Nontriviality means that the immersion in the family is not in the form of $r \circ \Phi$, where $r: \mathbb{R}_{1}^{3} \rightarrow \mathbb{R}_{1}^{3}$ is an immersion of $\mathbb{R}_{1}^{3}$. These kinds of surfaces are called timelike Bonnet surfaces by Chen and $\mathrm{Li}$ in [11]. Suppose that $\left\{e_{1}, e_{2}, e_{3}\right\}$ is a local orthonormal frame at the point $x \in M$, where $e_{1}$ is a timelike tangent vector, $e_{2}$ is a spacelike unit tangent vector, and $e_{3}$ is a spacelike unit normal vector field at $x \in M . e_{3}$ can be regarded as a map $e_{3}: M \rightarrow S_{1}^{2}$, where $S_{1}^{2}=\left\{x \in \mathbb{R}_{1}^{3}:\right.$ $\langle x, x\rangle=1\}$ is the de Sitter space. Let $w^{i}, 1 \leq i \leq 3$, be dual 1 -forms of $e_{i}$ defined by $w_{i}\left(e_{j}\right)=\left\langle e_{i}, e_{j}\right\rangle=\varepsilon_{i} \delta_{i j}, 1 \leq i, j \leq 3$, and let $w_{j}^{i}, 1 \leq i, j \leq 3$, be connection forms; then

$$
\begin{array}{r}
d x=w^{1} e_{1}+w^{2} e_{2}, \\
d e_{1}=w_{1}^{2} e_{2}+w_{1}^{3} e_{3},
\end{array}
$$




$$
\begin{aligned}
& d e_{2}=w_{2}^{1} e_{1}+w_{2}^{3} e_{3}, \\
& d e_{3}=w_{3}^{1} e_{1}+w_{3}^{2} e_{2},
\end{aligned}
$$

such that $w_{1}^{3}=w_{3}^{1}, w_{2}^{3}=-w_{3}^{2}$, and $w_{1}^{2}=w_{2}^{1}$.

The Weingarten map $A: T_{x} M \rightarrow T_{x} M$ is given by

$$
\begin{aligned}
& A e_{1}=-a e_{1}-b e_{2}, \\
& A e_{2}=b e_{1}-c e_{2},
\end{aligned}
$$

and $A$ has real eigenvector if and only if $(a+c)^{2} / 4-\left(a c+b^{2}\right) \geq$ 0 ; that is, $H^{2}-K=(a-c)^{2} / 4-b^{2} \geq 0$ [11]. Here, $H=(a+c) / 2$ and $K=a c+b^{2}$ are the mean and Gaussian curvatures of $M$, respectively.

Unless otherwise stated throughout this paper, we will assume that $H^{2}>K$ and $e_{1}, e_{2}$ are the eigenvectors. Thus, $b=0$ and

$$
\begin{aligned}
& w_{1}^{3}=-a w^{1}, \\
& w_{2}^{3}=c w^{2},
\end{aligned}
$$

where $a$ and $c$ are the principal curvatures throughout $e_{1}$ and $e_{2}$. Then, the mean and Gaussian curvatures of the surface $M$ are

$$
\begin{aligned}
& H=\frac{a+c}{2}, \\
& K=a c
\end{aligned}
$$

respectively, and $J=(a-c) / 2>0$. Suppose that $w_{1}^{2}=$ $h w^{1}+k w^{2}$; then $h$ and $k$ can be determined from the Cartan structure equations given by

$$
\begin{aligned}
& d w^{1}=w^{2} \wedge w_{2}^{1}, \\
& d w^{2}=w^{1} \wedge w_{1}^{2} .
\end{aligned}
$$

The Gaussian and Codazzi equations are

$$
\begin{aligned}
& d w_{1}^{2}=w_{1}^{3} \wedge w_{3}^{2}=-K w^{1} \wedge w^{2}, \\
& d w_{1}^{3}=w_{1}^{2} \wedge w_{2}^{3}, \\
& d w_{2}^{3}=w_{2}^{1} \wedge w_{1}^{3},
\end{aligned}
$$

respectively [11]. If we substitute the equations of (3) into the Codazzi equations, respectively, and make them equal to the exterior differentiations of (3), then we give

$$
\begin{aligned}
& \left(d a+(a-c) h w^{2}\right) \wedge w^{1}=0 \\
& \left(d c+(c-a) k w^{1}\right) \wedge w^{2}=0
\end{aligned}
$$

After reformulating the equations of (8), we can give

$$
\begin{aligned}
& d a=(c-a)\left(p w^{1}+h w^{2}\right), \\
& d c=(a-c)\left(k w^{1}+q w^{2}\right) .
\end{aligned}
$$

Since $d H=(d a+d c) / 2$, these last two equations give us

$$
2 d H=(a-c)\left((k-p) w^{1}+(q-h) w^{2}\right) .
$$

Here, if the functions $u$ and $v$ are defined as $u=k-p$ and $v=q-h$, then the last equation becomes

$$
2 d H=(a-c)\left(u w^{1}+v w^{2}\right)
$$

that is, $d H=J\left(u w^{1}+v w^{2}\right)$. Moreover, from (9), we get

$$
\begin{aligned}
\frac{d a}{a-c} & =(u-k) w^{1}-h w^{2}, \\
\frac{d c}{a-c} & =k w^{1}+(v+h) w^{2} .
\end{aligned}
$$

Thus, by the aid of these last two equations, we see

$$
d \ln (a-c)=(u-2 k) w^{1}-(v+2 h) w^{2} .
$$

If we take into consideration (11), then the gradient of the mean curvature function $H$ is

$$
\nabla H=\frac{a-c}{2}\left(-u e_{1}+v e_{2}\right) .
$$

Thus, it is easily seen that

$$
g(\nabla H, \nabla H)=\left(H^{2}-K\right)\left(-u^{2}+v^{2}\right) .
$$

Here, we assume that $\nabla H$ is nonnull vector field; that is, $\pm u \neq$ $v$. By taking $\varepsilon=\operatorname{sgn} g(\nabla H, \nabla H)= \pm 1$, we find

$$
\varepsilon\left(-u^{2}+v^{2}\right)=\frac{|g(\nabla H, \nabla H)|}{H^{2}-K} .
$$

By the fact that Hodge $*$ operator is defined as

$$
\begin{gathered}
* w^{1}=w^{2}, \\
* w^{2}=w^{1}, \\
*^{2}=1,
\end{gathered}
$$

the connection form $w_{1}^{2}$ given by (3) becomes

$$
* w_{1}^{2}=h * w^{1}+k * w^{2}=k w^{1}+h w^{2} .
$$

Let us define

$$
\begin{aligned}
& \theta^{1}=u w^{1}+v w^{2}, \\
& \theta^{2}=v w^{1}+u w^{2}, \\
& \alpha^{1}=u w^{1}-v w^{2}, \\
& \alpha^{2}=-v w^{1}+u w^{2} .
\end{aligned}
$$

Then, the following equations are obvious:

$$
\begin{aligned}
& * \theta^{1}=\theta^{2}, \\
& * \theta^{2}=\theta^{1}, \\
& * \alpha^{1}=\alpha^{2}, \\
& * \alpha^{2}=\alpha^{1} .
\end{aligned}
$$


By considering the second equation of (21), if we rearrange (11), we get

$$
2 d H=(a-c) \theta^{1} .
$$

In a similar way, from (18) and (20), (13) becomes

$$
d \ln (a-c)=\left(u w^{1}-v w^{2}\right)-2\left(k w^{1}+h w^{2}\right) .
$$

Thus, we find

$$
d \ln (a-c)=\alpha^{1}-2 * w_{1}^{2}
$$

\section{Timelike Bonnet Surfaces in Minkowski Space}

Since we will make use of it in the following section, let us briefly recall construction of the criterion given by [11] for a timelike surface to be a Bonnet surface in 3-dimensional Minkowski space.

Let $\bar{M}$ be another timelike surface in $\mathbb{R}_{1}^{3}$ with the principal direction vectors such that it is an isometric deformation of $M$ preserving the first fundamental form and principal curvatures. Suppose that $\left\{\bar{w}^{1}, \bar{w}^{2}\right\}$ is a principal coframe corresponding to orthonormal $\left\{\bar{e}_{1}, \bar{e}_{2}\right\}$ of $\bar{M}$; then the first fundamental form of $\bar{M}$ is

$$
-\left(\bar{w}^{1}\right)^{2}+\left(\bar{w}^{2}\right)^{2}=-\left(w^{1}\right)^{2}+\left(w^{2}\right)^{2},
$$

and the principal curvatures throughout $\bar{e}_{1}$ and $\bar{e}_{2}$ are

$$
\begin{aligned}
& \bar{a}=a, \\
& \bar{c}=c,
\end{aligned}
$$

respectively. It is seen from (26) that there is a function $\varphi$ on $\bar{M}$ such that

$$
\begin{aligned}
& \bar{w}^{1}=\cosh \varphi w^{1}+\sinh \varphi w^{2}, \\
& \bar{w}^{2}=\sinh \varphi w^{1}+\cosh \varphi w^{2} .
\end{aligned}
$$

By direct calculations and the exterior derivative of (28), we get $d \bar{w}^{1}=\bar{w}^{2} \wedge\left(-d \varphi+w_{1}^{2}\right)$ and $d \bar{w}^{2}=\bar{w}^{1} \wedge\left(-d \varphi+w_{1}^{2}\right)$. From the first Cartan structure equations, we obtain

$$
\bar{w}_{1}^{2}=\bar{w}_{2}^{1}=w_{1}^{2}-d \varphi .
$$

On the other hand, from (25), we write $d \ln (\bar{a}-\bar{c})=\bar{\alpha}^{1}-$ $2 * \bar{w}_{1}^{2}$. Equations (27) and (29) and the last equation give us $\alpha^{1}-2 * w_{1}^{2}=\bar{\alpha}^{1}-2 * \bar{w}_{1}^{2}$. If we apply $*$ operator to this equality, we find $* \alpha^{1}-2 w_{1}^{2}=* \bar{\alpha}^{1}-2 \bar{w}_{1}^{2}$. By the fact that $* \alpha^{1}=\alpha^{2}, * \alpha^{2}=\alpha^{1}, *^{2}=1$ we see $\alpha^{2}-2 w_{1}^{2}=\bar{\alpha}^{2}-2 \bar{w}_{1}^{2}$. After the necessary arrangements, we find $\left(\bar{w}_{1}^{2}-w_{1}^{2}\right)=(1 / 2)\left(\bar{\alpha}^{2}-\right.$ $\alpha^{2}$ ). If we consider (29), we get

$$
d \varphi=\frac{1}{2}\left(\alpha^{2}-\bar{\alpha}^{2}\right) .
$$

$2 d H=(\bar{\alpha}-\bar{c}) \bar{\theta}^{1}$ is satisfied for $\bar{M}$ with respect to (23). Considering (27) and comparing the last equation with (23), we see $\bar{\theta}^{1}=\theta^{1}$. Thus, $\bar{u} \bar{w}^{1}+\bar{v} \bar{w}^{2}=u w^{1}+v w^{2}$ and if we consider (28) we write

$$
\begin{aligned}
& \bar{u}=u \cosh \varphi-v \sinh \varphi, \\
& \bar{v}=-u \sinh \varphi+v \cosh \varphi
\end{aligned}
$$

By taking (20), if we substitute (28) and (31) into $\bar{\alpha}^{2}=-\bar{v} \bar{w}^{1}+$ $\bar{u} \bar{w}^{2}$, we obtain

$$
\bar{\alpha}^{2}=\sinh 2 \varphi \alpha^{1}+\cosh 2 \varphi \alpha^{2}
$$

Let us define $T=\operatorname{coth} \varphi$. The differentiation of $T$ is

$$
d T=T \alpha^{1}+\alpha^{2}
$$

The total differential equation (33) is satisfied by rotations of the principal directions with hyperbolic angles $\varphi$ during isometric deformations. The deformation is nontrivial if and only if (33) is completely integrable [11].

Theorem 1. Every timelike constant mean curvature surface $M$ with $H^{2}>K$ in $\mathbb{R}_{1}^{3}$ has one-parameter family of nontrivial isometric deformations preserving the mean curvature; that is, $M$ is timelike Bonnet surface [11].

In order to investigate the situations of $H$ being constant or nonconstant, let

$$
\begin{aligned}
& d \alpha^{1}=P \alpha^{1} \wedge \alpha^{2}, \\
& d \alpha^{2}=Q \alpha^{1} \wedge \alpha^{2},
\end{aligned}
$$

thus defining $P$ and $Q$. By substituting (34) into the exterior derivative of (33), we get

$$
(T P+Q-1) \alpha^{1} \wedge \alpha^{2}=0 .
$$

Thus, the following classification is satisfied:

$$
\begin{aligned}
& C_{1}: H=\text { constant. } \\
& C_{2}: H \neq \text { constant, } P=0, \text { and } Q=1 . \\
& C_{3}: H \neq \text { constant, } P \neq 0, \text { and } Q \neq 1 .
\end{aligned}
$$

If these categories are investigated separately, then $C_{1}, C_{2}$, and $C_{3}$ can be interpreted as follows.

Since the mean curvature is constant in the case of $C_{1}$ from (34) it is obvious that $u=v=0$. Thus, by (20), we see $\alpha^{1}=\alpha^{2}=0$. Consequently, from (33), $T$ is constant.

Since the mean curvature is nonconstant and $P=0$ and $Q=1$ in $C_{2}$, (35) is satisfied for all $T$.

The mean curvature is nonconstant and $P \neq 0$ and $Q \neq 1$ in $C_{3}$. In this case, by considering (35), we get

$$
T=\frac{1-Q}{P} .
$$

$T$ can be computed for any timelike surface with nonconstant mean curvature, nonnull $\nabla H$ and $H^{2}>K$ but in order to find 
the nontrivial isometry $\Phi$ preserving the mean curvature $T$ given in (36) must satisfy (33). Thus,

$$
d\left(\frac{1-Q}{P}\right)=\left(\frac{1-Q}{P}\right) \alpha^{1}+\alpha^{2}
$$

constitutes a criterion for being timelike Bonnet surface.

In the following section, we will check this criterion for timelike tangent developable surfaces and obtain the necessary and sufficient condition for timelike tangent developable surfaces to belong to case $C_{3}$.

\section{Timelike Tangent Developable Surfaces}

Timelike tangent developable surfaces can be investigated in two subcases when the curvature and torsion of base curve of these surfaces are nonconstant and constant.

4.1. Timelike Tangent Developable Surfaces with Base Curve with Nonconstant Curvature and Torsion. Let $M$ be a timelike tangent developable surface given by

$$
\begin{aligned}
X: I \times \mathbb{R} & \longrightarrow \mathbb{R}_{1}^{3} \\
(s, t) & \longrightarrow X(s, t)=\eta(s)+t e_{1}(s),
\end{aligned}
$$

where $\eta(s)$ is timelike curve parametrized by its arc-length $s$ and $e_{1}(s)=\dot{\eta}(s)$ is timelike unit tangent vector field. Also assume that $t>0$. (In the same way, $t<0$ gives the second sheet of this surface.) Let $e_{2}(s)$ be principal normal vector field of $\eta(s)$ and of course it is spacelike. Then, $\dot{e}_{1}(s)=$ $\kappa(s) e_{2}(s)$ such that $\kappa(s) \geq 0$ is the curvature of the base curve $\eta(s)$. From (38), it is seen that $X_{s}(s, t)=e_{1}(s)+t \kappa(s) e_{2}(s)$ and $X_{t}(s, t)=e_{1}(s)$. The first fundamental form of this surface is

$$
I=\left(-1+t^{2} \kappa^{2}(s)\right) d s^{2}-2 d s d t-d t^{2} .
$$

Then, $d X(s, t)=(d s+d t) e_{1}(s)+(t \kappa(s) d s) e_{2}(s)$ can be given from $d X(s, t)=X_{s} d s+X_{t} d t$. Also, keeping in mind $d X(s, t)=$ $w^{1} e_{1}(s)+w^{2} e_{2}(s)$, we see

$$
\begin{aligned}
& w^{1}=d s+d t, \\
& w^{2}=t \kappa(s) d s .
\end{aligned}
$$

By the fact that $w_{1}^{2}=g\left(d e_{1}(s), e_{2}(s)\right)$, we get $w_{1}^{2}=\kappa(s) d s$. On the other hand, since $w^{2}=t \kappa(s) d s$, that is, $\kappa(s) d s=w^{2} / t$, we find $w_{1}^{2}=(1 / t) w^{2}$. By defining $e_{3}(s)=e_{1}(s) \wedge e_{2}(s), e_{3}(s)$ is binormal vector field of base curve $\eta(s)$ and spacelike. Let us denote the torsion of $\eta(s)$ by $\tau(s)$. Considering the SerretFrenet formulae, we obtain

$$
\begin{aligned}
& w_{1}^{3}=g\left(d e_{1}(s), e_{3}(s)\right)=0=0 w^{1}, \\
& w_{2}^{3}=g\left(d e_{2}(s), e_{3}(s)\right)=-\tau(s) d s .
\end{aligned}
$$

Moreover, we get $w_{2}^{3}=-\tau(s)\left(w^{2} / t \kappa(s)\right)$ since $d s=w^{2} / t \kappa(s)$. From the fact that $\left\{w^{1}, w^{2}\right\}$ is the dual coframe corresponding to principal frame field $\left\{e_{1}, e_{2}\right\}$, the principal curvatures throughout the principal direction vector fields $a$ and $c$ satisfy $a=0>c=-\tau(s) / t \kappa(s)$. That is, since $t>0$, it is assumed that $\tau(s)>0$. By taking the principal curvatures in terms of curvature and torsion of base curve, $H$ and $J$ can be given as

$$
\begin{gathered}
H=\frac{1}{2}\left(\frac{-\tau(s)}{t \kappa(s)}\right), \\
J=\frac{1}{2}\left(\frac{\tau(s)}{t \kappa(s)}\right),
\end{gathered}
$$

respectively. On the other hand, from (11), we get

$$
d\left(\frac{-\tau(s)}{2 t \kappa(s)}\right)=\frac{1}{2} \frac{\tau(s)}{t \kappa(s)}\left(u w^{1}+v w^{2}\right) .
$$

If we substitute (40) into this last equation, we find

$$
d\left(\frac{-\tau(s)}{t \kappa(s)}\right)=\frac{\tau(s)}{t \kappa(s)}(u(d s+d t)+v(t \kappa(s)) d s) .
$$

If we call

$$
F(s)=\left(\ln \frac{\tau(s)}{\kappa(s)}\right)^{\prime}
$$

by considering (42) from (44) we obtain

$$
\begin{aligned}
& u=\frac{1}{t}, \\
& v=\frac{-F(s)}{t \kappa(s)}-\frac{1}{t^{2} \kappa(s)} .
\end{aligned}
$$

If we substitute (40) and (46) into (20), we get

$$
\begin{aligned}
\alpha^{1}= & \left(F(s)+\frac{2}{t}\right) d s+\frac{1}{t} d t, \\
\alpha^{2}= & \left(\kappa(s)+\frac{F(s)}{t \kappa(s)}+\frac{1}{t^{2} \kappa(s)}\right) d s \\
& +\left(\frac{F(s)}{t \kappa(s)}+\frac{1}{t^{2} \kappa(s)}\right) d t .
\end{aligned}
$$

The exterior differentiations of the equalities given in (47) are

$$
\begin{aligned}
& d \alpha^{1}=\frac{2}{t^{2}} d s \wedge d t \\
& d \alpha^{2} \\
& =\left(\frac{t^{2}\left(F^{\prime}(s)-F(s)(\ln \kappa(s))^{\prime}\right)+t\left(F(s)-(\ln \kappa(s))^{\prime}\right)+2}{t^{3} \kappa(s)}\right) d s \\
& \quad \wedge d t
\end{aligned}
$$

respectively. The exterior product of the equalities given in (47) is

$$
\alpha^{1} \wedge \alpha^{2}=\left(\frac{t^{2}\left(F^{2}(s)-\kappa^{2}(s)\right)+2 t F(s)+1}{t^{3} \kappa(s)}\right) d s
$$


such that it never vanishes. By putting (48) and (49) into the equalities in (34), we find

$$
P=\frac{2 t \kappa(s)}{t^{2}\left(F^{2}(s)-\kappa^{2}(s)\right)+2 t F(s)+1},
$$

Q

$$
=\frac{t^{2}\left(F^{\prime}(s)-F(s)(\ln \kappa(s))^{\prime}\right)+t\left(F(s)-(\ln \kappa(s))^{\prime}\right)+2}{t^{2}\left(F^{2}(s)-\kappa^{2}(s)\right)+2 t F(s)+1} .
$$

In order to check whether the criterion of being a timelike Bonnet surface given in the case of $C_{3}$ is satisfied or not, let us substitute the equations given in (50) into (36) and find

$$
T=\frac{t^{2}\left(F^{2}(s)-\kappa^{2}(s)-F^{\prime}(s)+F(s)(\ln \kappa(s))^{\prime}\right)+t\left(F(s)+(\ln \kappa(s))^{\prime}\right)-1}{2 t \kappa(s)} .
$$

By arranging (45), we get

$$
F(s)=(\ln \tau(s))^{\prime}-(\ln \kappa(s))^{\prime} .
$$

Thus, (51) becomes

$T$

$$
=\frac{t^{2}\left(-\kappa^{2}(s)-F^{\prime}(s)+F(s)(\ln \tau(s))^{\prime}\right)+t(\ln \tau(s))^{\prime}-1}{2 t \kappa(s)} .
$$

From exterior derivative of (53), we obtain

$$
\begin{aligned}
d T & =\left(\frac{t}{2 \kappa(s)}\left(-F^{\prime}(s)+F(s)(\ln \tau(s))^{\prime}-\kappa^{2}(s)\right)^{\prime}\right. \\
& -\frac{t}{2 \kappa^{2}(s)}\left(-F^{\prime}(s)+F(s)(\ln \tau(s))^{\prime}-\kappa^{2}(s)\right) \\
& +\frac{1}{2 \kappa(s)}(\ln \tau(s))^{\prime \prime}-\frac{t}{2 t \kappa^{2}(s)}(\ln \tau(s))^{\prime} \\
& \left.+\frac{1}{2 t \kappa^{2}(s)}\right) d s \\
& +\left(\frac{t}{2 \kappa(s)}\left(-F^{\prime}(s)+F(s)(\ln \tau(s))^{\prime}-\kappa^{2}(s)\right)\right. \\
& \left.+\frac{1}{2 t^{2} \kappa(s)}\right) d t
\end{aligned}
$$

In order to find the right side of (33), we consider (47) and (53) and find

$$
\begin{aligned}
T \alpha^{1}+\alpha^{2}= & \left(\frac{t^{2} F(s)\left(-F^{\prime}(s)+F(s)(\ln \tau(s))^{\prime}-\kappa^{2}(s)\right)+t\left(3 F(s)(\ln \tau(s))^{\prime}-2 F^{\prime}(s)\right)+F(s)+2(\ln \tau(s))^{\prime}}{2 t \kappa(s)}\right) d s \\
& +\left(\frac{t^{2}\left(-F^{\prime}(s)+F(s)(\ln \tau(s))^{\prime}-\kappa^{2}(s)\right)+t\left((\ln \tau(s))^{\prime}+2 F(s)\right)+1}{2 t^{2} \kappa(s)}\right) d t .
\end{aligned}
$$

If (54) and (55) are compared,

$$
(\ln \tau(s))^{\prime}=-2 F(s)
$$

is obtained since $d T=T \alpha^{1}+\alpha^{2}$. If we write this equation into (52), we get

$$
(\ln \tau(s))^{\prime}=-3 F(s) .
$$

If we substitute (56) into (52) and solve it, we find

$$
A \tau^{3}(s)=\kappa^{2}(s)
$$

where $A$ is a constant value such that $A \tau>0$. If (56) and (57) are written in (54),

$$
\begin{aligned}
d T= & \frac{t^{2}\left(\left(-F^{\prime}(s)-2 F^{2}(s)-\kappa^{2}(s)\right)^{\prime}+3 F(s)\left(-F^{\prime}(s)-2 F^{2}(s)-\kappa^{2}(s)\right)\right)-t\left(2 F^{\prime}(s)+6 F^{2}(s)\right)-3 F(s)}{2 t \kappa(s)} d s \\
& +\frac{t^{2}\left(F(s)(\ln \tau(s))^{\prime}-\kappa^{2}(s)-F^{\prime}(s)\right)+1}{2 t^{2} \kappa(s)} d t
\end{aligned}
$$


is obtained. By comparing (55) with (59) and substituting into (56), we get $\left(-F^{\prime}(s)-2 F^{2}(s)-\kappa^{2}(s)\right)^{\prime}+3 F(s)\left(-F^{\prime}(s)-2 F^{2}(s)-\right.$ $\left.\kappa^{2}(s)\right)=F(s)\left(-F^{\prime}(s)-2 F^{2}(s)-\kappa^{2}(s)\right)$. It is easily seen that

$$
\left(\frac{-F^{\prime}(s)-2 F^{2}(s)-\kappa^{2}(s)}{\tau(s)}\right)^{\prime}=0
$$

that is,

$$
\left(\frac{-F^{\prime}(s)-2 F^{2}(s)-\kappa^{2}(s)}{\tau(s)}\right)=B,
$$

where $B$ is constant. From this last equation, we get

$$
-F^{\prime}(s)-2 F^{2}(s)=\kappa^{2}(s)+B \tau(s) .
$$

If we put (56) and (58) into (62), then we find

$$
\tau^{\prime \prime}(s) \tau(s)-2\left(\tau^{\prime}(s)\right)^{2}=2 A \tau^{5}(s)+2 B \tau^{3}(s)
$$

By multiplying each side of the last equation by $2 / \tau(s)$, we get

$$
2 \tau^{\prime \prime}(s)-\frac{4}{\tau(s)}\left(\tau^{\prime}(s)\right)^{2}=4 A \tau^{4}(s)+4 B \tau^{2}(s)
$$

Here, assume that $d \tau / d s=\tau^{\prime}(s)=\sigma(\tau)$. Thus, (68) becomes

$$
\left(\sigma^{2}\right)^{\prime}-\frac{4}{\tau(s)} \sigma^{2}=4 A \tau^{4}(s)+4 B \tau^{2}(s)
$$

The solution of this differential equation is

$$
\sigma^{2}=\left(\tau^{\prime}\right)^{2}=4 A \tau^{5}+C \tau^{4}-4 B \tau^{3}, \quad C=\text { constant }
$$

Here, $\left(\tau^{\prime}\right)^{2} \geq 0$ and $A \tau^{3}(s)=\kappa^{2}(s)>0$. Thus, there is the inequality

$$
4 \tau^{2}+\frac{C}{A} \tau-4 \frac{B}{A} \geq 0 .
$$

If $\Delta=C^{2}-4(4 A)(-4 B)>0$, that is, $C^{2}>-64 A B$, then $\tau<r_{1}<r_{2}$ or $r_{1}<r_{2}<\tau$ and $\tau \neq 0$, where $r_{1}$ and $r_{2}$ are the roots of the quadratic trinomial $4 \tau^{2}+(C / A) \tau-4(B / A)=0$.

Thus, the following theorem can be given.

Theorem 2. Timelike tangent developable surface $X(s, t)$ is a timelike Bonnet surface if and only if

(i) $A \tau^{3}(s)=k^{2}(s), \quad A \tau(s)>0, A=$ constant,

(ii) $\tau^{\prime \prime}(s) \tau(s)-2\left(\tau^{\prime}(s)\right)^{2}=2 A \tau^{5}(s)+2 B \tau^{3}(s)$,

$B=$ constant or $\left(\tau^{\prime}\right)^{2}=A \tau^{3}\left(4 \tau^{2}+\frac{C}{A} \tau-4 \frac{B}{A}\right)$,

(iii) $C^{2}>-64 A B, \quad C=$ constant.
4.2. Timelike Tangent Developable Surfaces with Base Curve with Constant Curvature and Torsion. If the curvature and torsion of timelike base curve of timelike tangent developable surface $X(s, t)=\eta(s)+t e_{1}(s)$ are constants, then the base curve $\eta(s)$ is timelike circular helix. All $t$-constant curves are helices and $X(s, t)$ is a timelike flat helicoidal surface. Since $w^{1}, w^{2}, w_{1}^{2}, w_{1}^{3}, w_{2}^{3}, a$, and $c$ are determined as in Section 4.1, we have

$$
\begin{aligned}
F(s) & =0, \\
T & =\operatorname{coth} \varphi=\frac{-t^{2} \kappa^{2}-1}{2 t \kappa}, \\
\sinh \varphi & =\frac{2 t \kappa}{t^{2} \kappa^{2}-1}, \\
\cosh \varphi & =-\frac{t^{2} \kappa^{2}+1}{t^{2} \kappa^{2}-1},
\end{aligned}
$$

$$
\varphi \in(0, \infty)
$$

By considering the possibility of reflection, the forms of the image surface are

$$
\begin{aligned}
\bar{I} & =\left(-1+t^{2} \kappa^{2}(s)\right) d s^{2}-2 d s d t-d t^{2} \\
\bar{w}^{1} & = \pm\left(\cosh \varphi w^{1}+\sinh \varphi w^{2}\right) \\
& = \pm\left(d s+\frac{t^{2} \kappa^{2}+1}{t^{2} \kappa^{2}-1} d t\right) \\
\bar{w}^{2} & = \pm\left(\sinh \varphi w^{1}+\cosh \varphi w^{2}\right) \\
& = \pm\left(-t \kappa d s+\frac{2 t \kappa}{t^{2} \kappa^{2}-1} d t\right), \\
\bar{w}_{1}^{2} & = \pm\left(-d \varphi+w_{1}^{2}\right)= \pm\left(-\frac{2 \kappa}{t^{2} \kappa^{2}-1} d t+\kappa d s\right) \\
& = \pm \frac{1}{t} \bar{w}^{2}, \\
\bar{w}_{1}^{3} & =0 \bar{w}^{1}=0, \\
\bar{w}_{2}^{3} & =-\frac{\tau(s)}{t \kappa(s)} \bar{w}^{2}= \pm\left(\tau d s-\frac{2 t \kappa}{t^{2} \kappa^{2}-1} d t\right) .
\end{aligned}
$$

Moreover, the image surface $\Phi(X(s, t))$ is flat but it is not a cylinder or a cone because of $d \alpha^{1}=0$; that is, $P=0$ is satisfied for cylinders and cones. Thus, a timelike developable surface cannot be a cylinder or a cone. Therefore, image surface is timelike developable surface given by $Y(\widetilde{s}, \widetilde{t})=\widetilde{\eta}(\widetilde{s})+\widetilde{t} \widetilde{e}_{1}(\widetilde{s})$, $\tilde{t}>0$. Here, $\widetilde{s}=-s-(2 / \kappa) \operatorname{arctanh}(\kappa t)$ is the arc-length of the timelike base curve $\widetilde{\eta}(\widetilde{s})$ and $\dot{\tilde{\eta}}(\widetilde{s})=\widetilde{e}_{1}(\widetilde{s})$ is timelike unit tangent vector. The forms of timelike surfaces $Y(\widetilde{s}, \widetilde{t})$ are

$$
\begin{aligned}
\widetilde{I} & =\left(-1+\widetilde{t}^{2} \widetilde{\kappa}^{2}(\widetilde{s})\right) d \widetilde{s}^{2}-2 d \widetilde{s} d \widetilde{t}-d \widetilde{t} \\
\widetilde{w}^{1} & =d \widetilde{s}+d \widetilde{t}, \\
\widetilde{w}^{2} & =\widetilde{t} \widetilde{\kappa}(\widetilde{s}) d \widetilde{s},
\end{aligned}
$$




$$
\begin{aligned}
& \widetilde{w}_{1}^{2}=\widetilde{\kappa}(\widetilde{s}) d \widetilde{s}=\frac{\widetilde{w}^{2}}{\widetilde{t}}, \\
& \widetilde{w}_{1}^{3}=0 \widetilde{w}^{1}, \\
& \widetilde{w}_{2}^{3}=-\frac{\widetilde{\tau}(\widetilde{s})}{\widetilde{t} \widetilde{\kappa}(\widetilde{s})} \widetilde{w}^{2} .
\end{aligned}
$$

By comparing the forms given in (70) with (71), one can see that $\bar{w}^{1}$ should be taken with the sign + whereas $\bar{w}^{2}$ should be taken with the sign -; that is, the reflection is necessary and $\tilde{t}=t, \widetilde{\kappa}=\kappa, \widetilde{\tau}=\tau, \widetilde{s}=-s-(2 / \kappa) \operatorname{arctanh}(\kappa t)$. Thus, the image surface is the original surface and the nontrivial isometry preserving the mean curvature is

$$
\begin{aligned}
\Phi: X(s, t) & \longrightarrow X(s, t) \\
(s, t) & \longrightarrow \Phi(s, t)=\left(-s-\frac{2}{\kappa} \operatorname{arctanh}(\kappa t), t\right)
\end{aligned}
$$

or

$$
\begin{aligned}
\Phi\left(\eta(s)+t e_{1}(s)\right)= & \eta\left(-s-\frac{2}{\kappa} \operatorname{arctanh}(\kappa t)\right) \\
& +t e_{1}\left(-s-\frac{2}{\kappa} \operatorname{arctanh}(\kappa t)\right) .
\end{aligned}
$$

Thus, the following corollaries can be given.

Corollary 3. The mapping $\Phi: X(s, t) \rightarrow X(s, t)$ is a timelike isometry preserving the mean curvature. The second fundamental forms of corresponding surfaces are different; that is, $\Phi$ is nontrivial.

Corollary 4. $\widetilde{s}=-s-(2 / \kappa) \operatorname{arctanh}(\kappa t), \tilde{t}=-t<0$, is also a timelike nontrivial isometry from $X$ to its other sheet preserving mean curvature.

\section{Conflict of Interests}

The authors declare that there is no conflict of interests regarding the publication of this paper.

\section{References}

[1] O. Bonnet, "Mémoire sur la théorie des surfaces applicables," Journal de l'École Polytechnique, vol. 42, pp. 72-92, 1867.

[2] E. Cartan, "Sur les couples de surfaces applicables avec conservation des courbures principales," Bulletin des Sciences Mathématiques, vol. 66, no. 2, pp. 55-72, 74-85, 1942.

[3] H. B. Lawson, "Complete minimal surface in $S^{3}$," Annals of Mathematics, vol. 92, no. 2, pp. 335-374, 1970.

[4] S.-S. Chern, "Deformation of surfaces preserving principal curvatures," in Differential Geometry and Complex Analysis, H. E. Rauch Memorial Volume, pp. 155-163, Springer, Berlin, Germany, 1985.

[5] I. M. Roussos, “The helicoidal surfaces as Bonnet surfaces," The Tohoku Mathematical Journal. Second Series, vol. 40, no. 3, pp. 485-490, 1988.
[6] I. M. Roussos, "Tangential developable surfaces as Bonnet surfaces," Acta Mathematica Sinica, vol. 15, no. 2, pp. 269-276, 1999.

[7] I. M. Roussos, "Global results on Bonnet surfaces," Journal of Geometry, vol. 65, no. 1-2, pp. 151-168, 1999.

[8] Z. Soyuçok, "The problem of non-trivial isometries of surfaces preserving principal curvatures," Journal of Geometry, vol. 52, no. 1-2, pp. 173-188, 1995.

[9] Z. Soyuçok, "The problem of isometric deformations of a Euclidean hypersurface pre-serving mean curvature," Bulletin of the Technical University of Istanbul, vol. 49, no. 3-4, pp. 551-562, 1996.

[10] H. Bağdatli and Z. Soyuçok, "On the problem of isometry of a hypersurface preserving mean curvature," Proceedings Mathematical Sciences, vol. 117, no. 1, pp. 49-59, 2007.

[11] W. Chen and H. Li, "On the classification of the timelike Bonnet surfaces," in Geometry and Topology of Submanifolds 10, Differential Geometry in Honor of Professor S S Chern, pp. 18-31, Peking University, Beijing, China; TU Berlin, Berlin, Germany, 1999. 


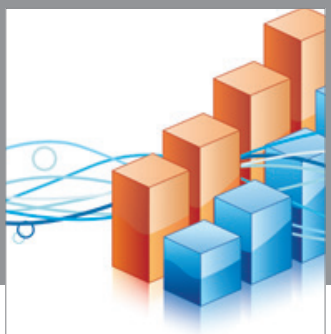

Advances in

Operations Research

vatem alat4

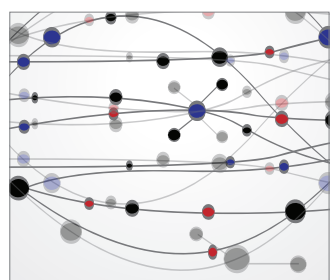

\section{The Scientific} World Journal

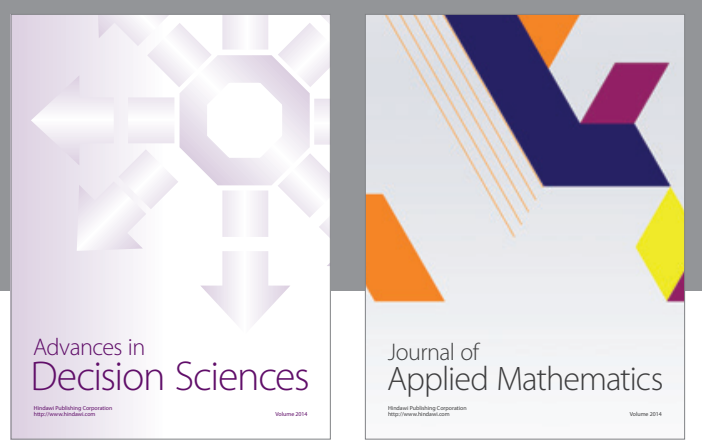

Algebra

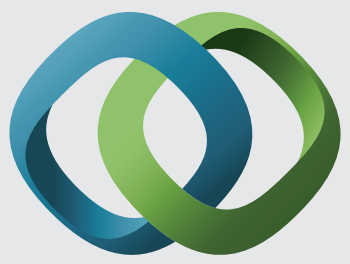

\section{Hindawi}

Submit your manuscripts at

http://www.hindawi.com
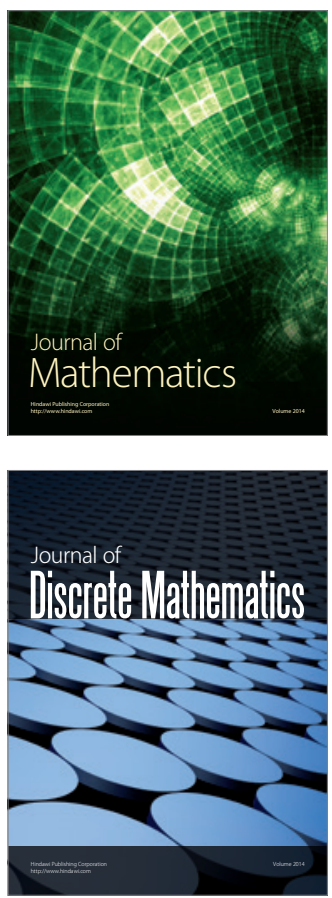

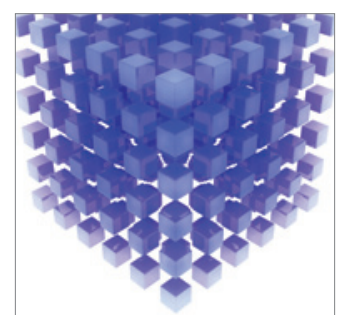

Mathematical Problems in Engineering


Journal of

Function Spaces

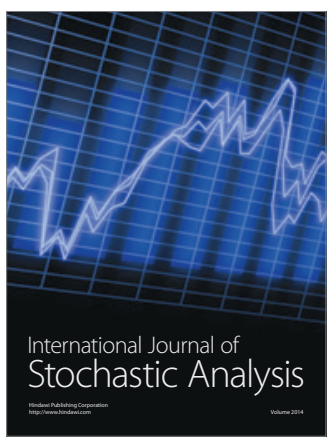

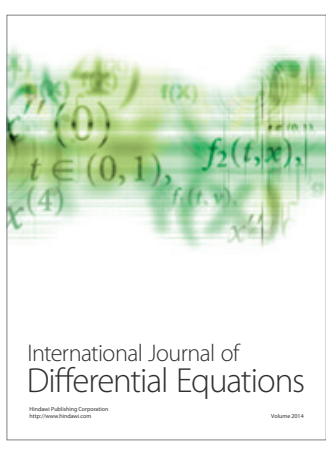
